1990-03-01

\title{
Photoluminescence Studies in ZnxCd1-xTe Single Crystals
}

\author{
David D. Allred \\ allred@byu.edu \\ Jesus González-Hernández \\ Elías López-Cruz \\ Worth P. Allred \\ allred@byu.edu
}

Follow this and additional works at: https://scholarsarchive.byu.edu/facpub

Part of the Astrophysics and Astronomy Commons, and the Physics Commons

\section{Original Publication Citation}

The following article appeared in J. González-Hernández, Elías López-Cruz, D.D. Allred, and W.P. Allred, "Photoluminescence Studies in ZnxCd1-xTe Single Crystals," Journal of Vacuum Science \& Technology A 8, 325559 (199). and can be found at [http://avspublications.org/jvsta/ resource/1/jvtad6/v8/i4/p3255_s1][http://dx.doi.org/1.1116/1.576574].

\section{BYU ScholarsArchive Citation}

Allred, David D.; González-Hernández, Jesus; López-Cruz, Elías; and Allred, Worth P., "Photoluminescence Studies in ZnxCd1-xTe Single Crystals" (1990). Faculty Publications. 1196.

https://scholarsarchive.byu.edu/facpub/1196

This Peer-Reviewed Article is brought to you for free and open access by BYU ScholarsArchive. It has been accepted for inclusion in Faculty Publications by an authorized administrator of BYU ScholarsArchive. For more information, please contact ellen_amatangelo@byu.edu. 


\section{Photoluminescence studies in $\mathrm{Zn}_{x} \mathrm{Cd}_{1-x}$ Te single crystals}

J. González-Hernándeza)

Departamento de Física, Centro de Investigación y de Estudios Avanzados del IPN, 07000, México, D.F.

Elias López-Cruz

Departamento de Física del Instituto de Ciencias, Universidad Autónoma de Puebla, Apdo. J.48 Puebla 72570

Pue., México

D.D. Allred

Physics and Astronomy Department, Brigham Young University, Provo, Utah 84602

Worth P. Allred

Galtech Semiconductor Materials Corporation, Mt. Pleasant, Utah 84647

(Received 10 July 1989; accepted 17 February 1990)

The crystalline quality of $\mathrm{Zn}_{x} \mathrm{Cd}_{1-x}$ Te single crystals prepared by a modified Bridgman method with $0 \leqslant x \leqslant 0.05$ has been analyzed using photoluminescence. The spectrum of a typical sample is dominated by lines originating from the recombination of free and bound excitons. Lines due to free excitons in their ground and first excited states are observed in both the pure $\mathrm{CdTe}$ and the mixed crystals. Excitons bound to $\mathrm{Cd}$ vacancies are observed in the pure CdTe crystal but not in the mixed crystal. Weaker and broader features appearing at energies below the exciton emission range were associated with transitions involving free-to-bound and bound-to-bound levels. The origin of the various lines in the spectra was deduced from the detailed measurements of the dependence of the spectrum on temperature and excitation intensity.

\section{INTRODUCTION}

Large diameter cadmium telluride (CdTe) and zinc cadmium telluride $\left(\mathrm{Zn}_{x} \mathrm{Cd}_{1-x} \mathrm{Te}\right)$ single crystals are the substrates of choice for epitaxial growth of mercury cadmium telluride $\left(\mathrm{Hg}_{y} \mathrm{Cd}_{1-y} \mathrm{Te}\right)$ layered structures. ${ }^{1-3}$ Devices based on the latter materials are assuming increasing importance for a number of device applications such as photovoltaic and photoconductive infrared detectors. The quality of the epitaxial layers is critically dependent on the nature and quality of the substrate. A reduction of the defect density in overgrown layers has been achieved by using $\mathrm{Zn}_{x} \mathrm{Cd}_{1-x} \mathrm{Te}$ $(x=0.04)$ substrates. ${ }^{4,5}$ Since the lattice parameters of the mixed compound with $x \sim 0.04$ approximately lattice-match epitaxial growth of $\mathrm{Hg}_{y} \mathrm{Cd}_{1-y} \mathrm{Te}$ for all values of $y$, the density of defects due to misfit dislocation is minimized. Furthermore, because defects in the substrate often propagate up to the epitaxial layers, the number of substrate defects must be also minimized. Likewise, the substrate must be very pure since a substrate with high impurity levels can serve as a source of contamination to the epitaxial layers during growth and subsequent thermal processing.

Several characterization techniques have been used to study crystalline perfection in II-VI compounds. ${ }^{6-8}$ Photoluminescence (PL) $)^{9,10}$ and cathodoluminescence ${ }^{10}$ have been employed extensively to characterize defects and impurities in CdTe. However, because the interest in $\mathrm{Zn}_{x} \mathrm{Cd}_{1-x} \mathrm{Te}$ has developed only in the last few years, few publications have appeared analyzing the PL spectra of the mixed compound. ${ }^{11-13}$ The studies in CdTe led to a qualitative model indicating that the ratio of two prominent lines in the PL spectrum, the first related to excitons and the other associated with recombination processes involving defect levels, is a good measure of the quality of CdTe. ${ }^{6,14}$

In this paper, we have used PL to analyze the crystalline quality of $Z_{x} \mathrm{Cd}_{1-x} \mathrm{Te}$ single crystals with $0 \leqslant x \leqslant 0.05$. The PL spectra show intense emission due to free and bound exciton recombination and no emission associated with crystal imperfection and deeper impurity levels. The origin of the various lines in the spectra was deduced from detailed measurements of the dependence of the spectra on temperature and laser intensity.

\section{EXPERIMENTAL}

Large area ( 3 in diam) $\mathrm{CdTe}$ and $\mathrm{Zn}_{x} \mathrm{Cd}_{1-x}$ Te samples were grown from the melt by a modified Bridgman method at Galtech Semiconductor Materials Corporation. The compositions of the $\mathrm{Zn}_{x} \mathrm{Cd}_{1-x} \mathrm{Te}$ samples from $x=0$ to $x=0.05$ were determined from electron microprobe analysis. The composition was also determined from the energy of the main exciton line observed by $\mathrm{PL}$ at $12 \mathrm{~K} .^{11}$ All crystals were unintentionally doped, and have an approximate roomtemperature resistivity of $5 \times 10^{6} \Omega \mathrm{cm}$ for $\mathrm{Zn}_{x} \mathrm{Cd}_{1-x}$ Te and $8 \times 10^{8} \Omega \mathrm{cm}$ for CdTe. For this study, all samples were cleaved along the (110) surface in an Ar atmosphere and the PL measurement was done immediately after the cleaving procedure without exposing the cleaved surface to atmospheric contaminations. The above mentioned procedure, previous to measurement, was adopted because, we notice that long exposure to air might change the relative intensity of the observed emission lines in the spectra. For the PL measurements, the crystals were placed in a variable-temperature helium gas closed-cycle refrigerator where the temperature can be stabilized within $\pm 0.2 \mathrm{~K}$ over the range of 11-300 K. As excitation sources, we used $\mathrm{He}-\mathrm{Ne}$ and Ar ion lasers. The laser power impinging on the sample ranged from 0.1-5 $\mathrm{mW}$ with the laser beam focused using cylindrical optics into a vertical line-shaped spot with approximate dimensions of $0.05 \times 0.5 \mathrm{~cm}^{2}$. The PL light was analyzed with a 
double monochromator, whose slits were set to achieve a resolution of less than $1 \AA$. Light was detected by a Hamamatsu model R636 photomultiplier tube coupled to a lockin amplifier system. The tube is used at room temperature and has an $\mathbf{S 2 0}$ spectral response curve.

\section{RESULTS AND DISCUSSION}

Figure 1 shows a typical $12 \mathrm{~K}$ PL spectra recorded between 755 and $880 \mathrm{~nm}$ for a $\mathrm{Zn}_{x} \mathrm{Cd}_{1-x} \mathrm{Te}(x=0.04)$ and for a pure CdTe cleaved surface. Both spectra are plotted with the same abscissa. Considering the origin of the emission lines, the PL spectra in Fig. 1 can be divided in three regions: (a) the exciton region, for wavelengths $\lambda$ lower than about 775 and $785 \mathrm{~nm}$ for the mixed and pure crystals respectively, (b) the free-to-bound and bound-to-bound level transitions for intermediate $\lambda$, and (c) emission at $\lambda>820$ nm associated with crystal imperfections and deeper impurity levels. ${ }^{6,15}$ In the first region, emission lines due to free and bound exciton recombination are observed. In the intermediate emission range, the PL spectrum of pure CdTe crystals shows a well defined doublet at $795.5 \mathrm{~nm}(1.5588 \mathrm{eV})$ and $798 \mathrm{~nm}(1.5539 \mathrm{eV})$, denoted in Fig. 1 by F-A and D-A, respectively. The origin of this doublet will be discussed later in this section. The doublet in our data is replicated at energies of approximately $21.5 \mathrm{meV}$, the energy of a LO-phonon, below the first two peaks. Thus, the structure at about 810 and $820 \mathrm{~nm}$ are attributed to the first and second order phonon replicas of the main doublet.

The spectrum of a typical $\mathrm{Zn}_{x} \mathrm{Cd}_{1-x}$ Te single crystal with $x=0.04$, is qualitatively similar to that of pure CdTe prepared under similar conditions (Fig. 1). The main differences between the two spectra being the downward wavelength shift in all emission lines and broader bound exciton lines in the mixed crystal. The former is due to the wider band gap and the latter to statistical fluctuations in the distribution of cation atoms, $\mathrm{Zn}$ and $\mathrm{Cd}$ in our case. ${ }^{16}$ Notice that both spectra of Fig. 1 do not show any significant emission for $\lambda \gtrsim 820 \mathrm{~nm}$ which, according to many other reports, is an indication of high crystalline quality. ${ }^{6,9,10,14}$ The emission intensities of the different lines in both spectra are very

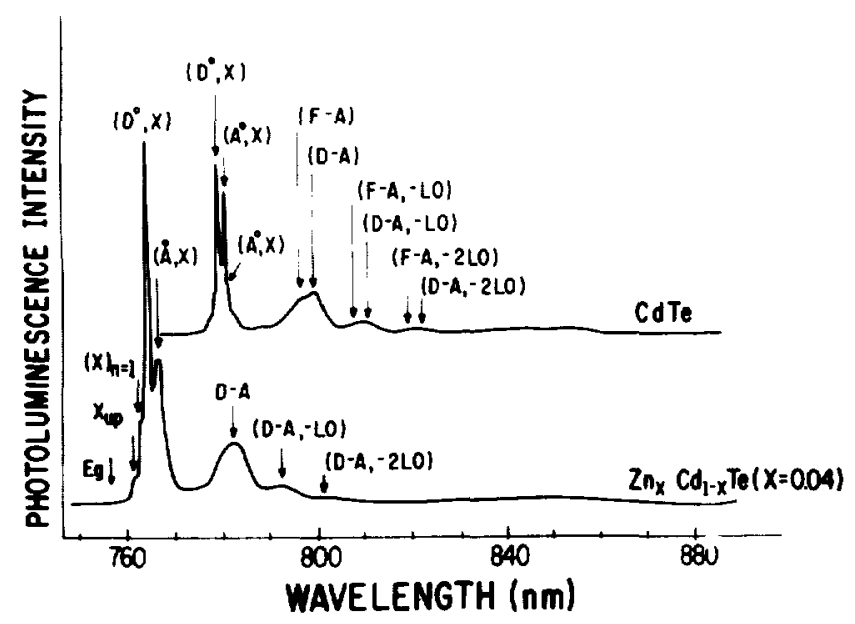

Fig. 1. PL spectra for a $\mathrm{Zn}_{x} \mathrm{Cd}_{1},{ }_{x} \mathrm{Te}(x=0.04)$ and for a pure CdTe cleaved surfaces. similar for the same excitation power.

Figure 2 shows the expanded spectra of the exciton region for the same two samples as in Fig. 1. Free excitons recombine with the emission of photons with an energy $10.5 \mathrm{meV}$ less than the band gap which, at $12 \mathrm{~K}$, is at $772 \mathrm{~nm}(1.606$ $\mathrm{eV})$ and $757.6 \mathrm{~nm}(1.6368 \mathrm{eV})$ for pure CdTe and the mixed crystal, respectively. Thus, the emission line due to recombination of free excitons in their ground state, denoted by $(X)_{n=1}$, in Fig. 2, appears at $777.2 \mathrm{~nm}(1.5955 \mathrm{eV})$ for $\mathrm{CdTe}$ and $762.5 \mathrm{~nm}(1.6262 \mathrm{eV})$ for the mixed crystal, respectively. The emission line due to the recombination of excitons in their first excited state have been reported only in a few high-quality CdTe samples ${ }^{13,17,18}$ and to our knowledge, it has never been reported before in the mixed crystals. The lines denoted by $(X)_{n=2}$ in both spectra of Fig. 2, correspond to these transitions, indicating that in these samples the free excitons have a longer lifetime than in previously studied samples. ${ }^{11-13}$ The longer exciton lifetime is an indication of high crystalline perfection. The lines denoted by $(X)_{\text {up }}$ in Fig. 2 have been previously identified with the upper branch of the exciton-polariton interaction. ${ }^{19}$

Free excitons can get trapped at crystalline imperfections such as impurities and structural defects thereby becoming bound excitons. The two main lines at $778.3 \mathrm{~nm}(1.5932 \mathrm{eV})$ and $779.5 \mathrm{~nm}(1.5907 \mathrm{eV})$ denoted by $\left(D^{\circ}, X\right)$ and $\left(A^{\circ}, X\right)$ in the spectrum of the pure CdTe crystal are assigned to the recombination of excitons bound to neutral donors and to neutral acceptors, respectively. The identification of these lines is based on a variety of evidences: (i) The exciton binding energy of 2.3 and $5 \mathrm{meV}$ obtained from the spectra for the $\left(D^{\circ}, X\right)$ and $\left(A^{\circ}, X\right)$, respectively, is close to the 3 and $7 \mathrm{meV}$ calculated for excitons bound to donors and acceptors in CdTe, respectively; ${ }^{20}$ (ii) The $778.3 \mathrm{~nm}$ line occurs in the wavelength range where $\left(D^{0}, X\right)$ recombination has been seen $^{21}$ due to shallow hydrogenic donors; (iii) PL emission at $77,9.5$ and $780 \mathrm{~nm}$ has been previously attributed to $\left(A^{0}, X\right)$ recombination in CdTe crystals; ${ }^{22,23}$ and (iv) the intensity of the $779.5 \mathrm{~nm}$ drastically decreases upon annealing at $600^{\circ} \mathrm{C}$ for $1 \mathrm{~h}$ under Cd-saturated atmosphere. This latter result suggests that the $\left(A^{0}, X\right)$ line is related to the

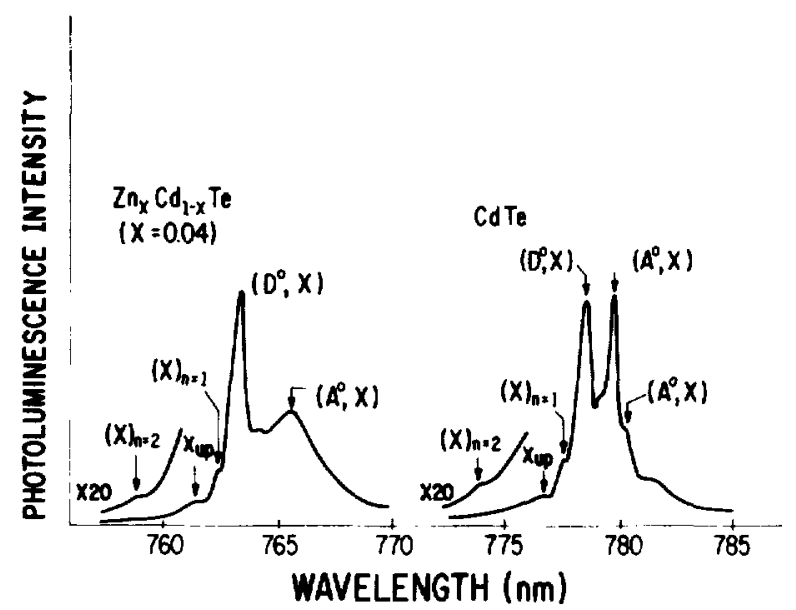

FIG. 2. Expanded spectra of the exciton region for the two cleaved surfaces in Fig. 1. 
recombination of excitons trapped at $\mathrm{Cd}$ vacancies complex as has been previously reported by Seto $\mathrm{et}_{\mathrm{al}}{ }^{24}$

In the spectrum of the $\mathrm{Zn}_{x} \mathrm{Cd}_{1-x} \mathrm{Te}$ crystal, in Fig. 2, the lines at $763.5 \mathrm{~nm}(1.624 \mathrm{eV})$ and $765.8 \mathrm{~nm}(1.6191 \mathrm{eV})$ have been identified with $\left(D^{\circ}, X\right)$ and $\left(A^{\circ}, X\right)$ transitions, respectively. The assignment for the line at $763.5 \mathrm{~nm}$ is based on the fact that its exciton binding energy of $2.3 \mathrm{eV}$ is the same found for the $\left(D^{0}, X\right)$ line in the pure CdTe crystal. On the other hand, the exciton binding energy of $7.1 \mathrm{meV}$ obtained for the line at $765.8 \mathrm{~nm}$ is closed to the predicted $7 \mathrm{meV}$ for a $\left(A^{0}, X\right)$ transition. This latter assignment is also in agreement with previous observations in other alloyed compound semiconductors showing that the $\left(A^{0}, X\right)$ lines are much more sensitive to alloying and the linewidth increases at a rate much greater than that of the $\left(D^{0}, X\right)$ lines. ${ }^{16}$ It is worthy to mention that annealing under $\mathrm{Cd}$ atmosphere results in almost no change in the PL spectrum of the $\mathrm{Zn}_{x} \mathrm{Cd}_{1-x} \mathrm{Te}$ crystals. This suggest that the $\left(A^{0}, X\right)$ line in the mixed crystal is probably not related with exciton recombination in volving $\mathrm{Cd}$ vacancy levels. This result is in agreement with the observation indicating that $\mathrm{Zn}$ addition to $\mathrm{CdTe}$ improves crystal quality, ${ }^{25,26}$ in particular reducing the density of $\mathrm{Cd}$ vacancies.

Figures 3 and 4 respectively, show the PL spectra of a pure $\mathrm{CdTe}$ and a $\mathrm{Zn}_{x} \mathrm{Cd}_{1-x} \mathrm{Te}(x=0.03)$ crystals recorded at various temperatures. In both figures, all peaks related with the recombination of free and bound excitons move to longer wavelengths (lower energies) and their intensities decrease with increasing temperature. The peak shift is due to the shrinkage of the band gap energy with increasing temperature while the rate of the intensity decrease depends on the process involved. The fact that the line at $778.3 \mathrm{~nm}$, in the spectrum of the CdTe crystal, exist at higher temperatures is possibly due to the superposition of donor-valence band $(D, h)$ recombination with the $(D, X) .{ }^{27}$ Also notice that the temperature dependence of the $779.5 \mathrm{~nm}$ line is consistent with the $\left(A^{0}, X\right)$ identification because the PL intensity of acceptor-bound excitons decreases very rapidly with increasing temperature.

Between 790 and $800 \mathrm{~nm}$ for CdTe (Fig. 3) and 780 and $790 \mathrm{~nm}$ for the mixed crystal (Fig. 4), both spectra show a broad feature. In the case of CdTe, the peak has two distinct humps while for $\mathrm{Zn}_{x} \mathrm{Cd}_{1-x}$ Te only one hump can be seen. In CdTe the longer wavelength feature is dominant at the lowest temperatures but decreases rapidly with increasing temperature and disappears above $30 \mathrm{~K}$. Its companion at shorter wavelengths in turn disappears at higher temperatures. This result agrees with previous studies in which the former peak has been assigned to $(D-A)$ and the latter peak to $(F-A)$ transitions. ${ }^{14}$ The deconvolution of the double hump into two Gaussian lines is shown in Fig. 3. There is no observed movement of the $(D-A)$ line position with increasing temperature, contrary to what is expected for a $(D, A)$ transition

As mentioned, the wide band at about $784.8 \mathrm{~nm}(1.58 \mathrm{eV})$ in the 11.5 K PL spectrum of the mixed crystal (Fig. 4) does not show a well-defined double-hump structure. However, as can be seen from that figure, as the temperature increases that band first moves to shorter wavelengths, reaching a

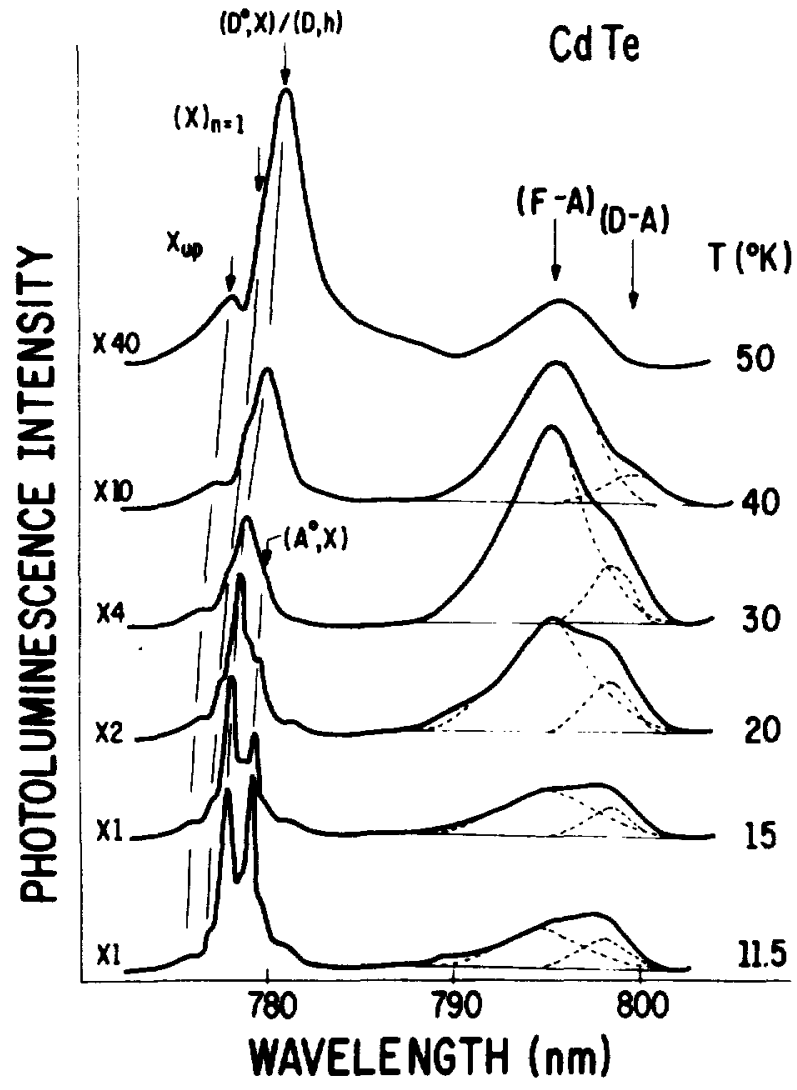

FIG. 3. PL spectra of a cleaved CdTe surface recorded at various temperatures.

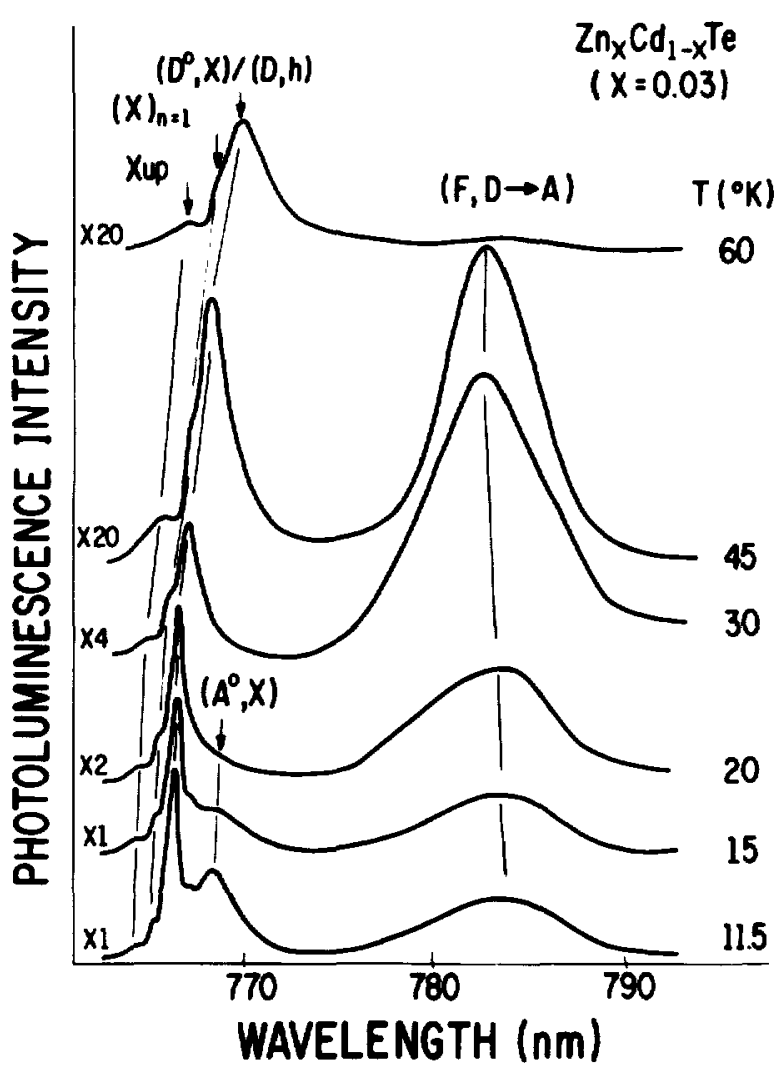

FIG. 4. PL spectra of a $\mathrm{Zn}_{x} \mathrm{Cd}_{1-x} \mathrm{Te}(x=0.03)$ cleaved surfaces recorded at various temperatures. 
maximum shift at about $30 \mathrm{~K}$ and then moves toward longer wavelengths for higher temperatures. The position of this band is shown as a function of sample temperature in Fig. 5 . For comparison, the figure also shows similar data for the $\left(D^{0}, X\right)$ line. Contrary to the broad emission band, the bound exciton transition decreases monotonically following the shrinkage of the band gap. Knowing that the position of an $(F-A)$ transition must also track the band gap shrinkage with temperature, we must conclude that the broadband contains at low temperatures two transitions. In analogy with what we learned for our CdTe samples, we conclude that at temperatures above $40 \mathrm{~K}$ the $(F-A)$ transition is dominant whereas for lower temperatures the $(D-A)$ transition is predominantly observed. Similar to the pure $\mathrm{CdTe}$ crystal, at higher temperatures, the $(F-A)$ transition appears to dominate the PL emission in that region of the spectrum. This assignment is in disagreement with previous studies where this transition has been attributed purely to $F$ $A$ even at low temperatures. " Our assignment is further supported by the data in Fig. 6, which shows the energy position of this band at two different temperatures as a function of the intensity of the excitation. As a reference, the figure also shows data for the $\left(D^{0}, X\right)$ band. As can be seen from this figure, at lower temperatures $(11.5 \mathrm{~K})$ the position of that band moves to higher energies for increasing excitation intensities, as one expects for a $(D-A)$ transition. Now, if the sample temperature is $60 \mathrm{~K}$, no significant shift is observed, as expected for an $(F-A)$ transition. The observed energy shift of about $4.2 \mathrm{meV}$ for two orders of magnitude change in the excitation intensity, is of the same order as that usually observed (5-6 meV) for (D-A) pair recombination in GaAs. ${ }^{28}$ From the difference between the band-gap energy and the conduction band to acceptor transition, we calculate a value of 45 and $48 \mathrm{meV}$ for the ionization energy of acceptor level for the pure and mixed crystals, respectively.

\section{CONCLUSIONS}

High resistivity intrinsic $\mathrm{Zn}_{x} \mathrm{Cd}_{1-x}$ Te single crystals prepared by a modified Bridgman method have been studied

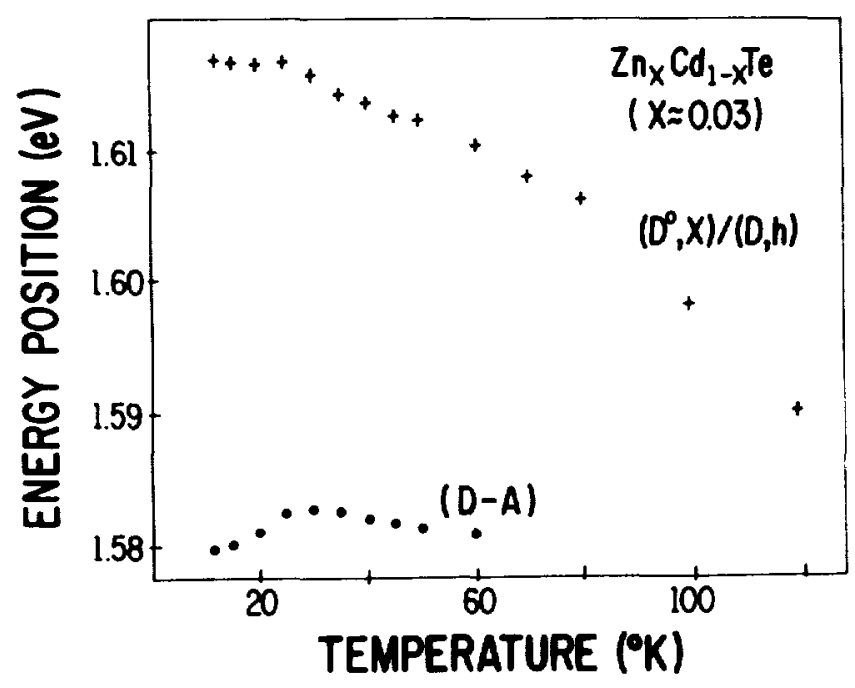

FIG. 5. Position of the $\left(D^{\circ}, X\right) /(D, h)$ and $(D-A)$ lines as a function of temperatures for $\mathrm{Zn}_{x} \mathrm{Cd}_{1} \times \mathrm{Te}(x=0.03)$.

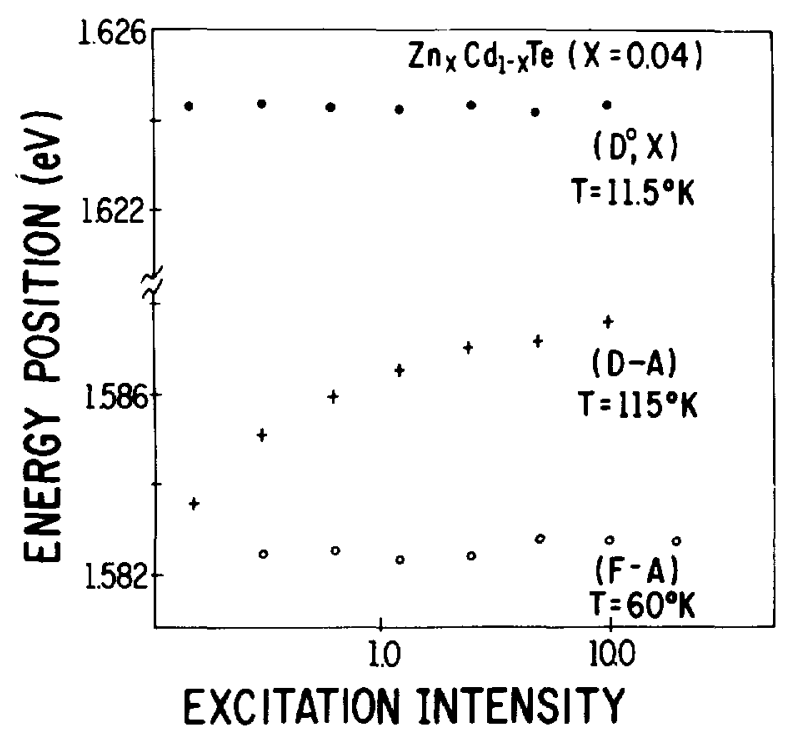

Fig. 6. Position of the $\left(D^{\circ}, X\right)$ and $(D-A)$ transition at 11.5 and of the $(F-$ $A$ ) transition at $60 \mathrm{~K}$ as a function of the intensity of the excitation.

using PL. The PL spectra indicate a high crystalline perfection for all measured samples in the compositional range investigated. As a result of that, the PL spectra show lines due to the recombination of free excitons in their ground and first excited state levels, and no emission associated with structural and/or deep impurity levels. In the low temperature $(T<30 \mathrm{~K})$ PL spectra of the mixed crystals, the broad emission band about $40 \mathrm{meV}$ below the excitonic region has been identified as due to donor-acceptor $(D-A)$ transitions rather than to free electron-acceptor transitions, as previously reported. ${ }^{11}$ The assignments for the various lines in the spectra were made from detailed measurements of the dependence of the spectrum on temperature and excitation laser intensity. In addition, annealing of all samples under saturated $\mathrm{Cd}$ atmosphere were perform to assist in the correct identification of the lines in the spectra. The PL spectra also show evidence for improved crystalline quality due to the $\mathrm{Zn}$ incorporation.

*) Partially supported by Brigham Young University

' J. J. Kennedy, P. M. Amirtharaj, P. R. Boyd, and S. B. Qadri, J. Cryst. Growth 86, 93 (1988).

${ }^{2}$ J. D. Murphy, Proc. SPIE 659, 2 (1986).

${ }^{3}$ A. M. Glass, R. D. Feldman, D. W. Kisker, P. M. Bridenbaugh, and P. M. Mankiewich, Proc. SPIE 659, 142 (1986).

${ }^{4}$ R. D. Feldman, R. F. Austin, A. H. Dayem, and E. H. Westerwick, Appl. Phys. Lett. 49, 797 (1986).

'R. H. Miles and T. C. McGill, J. Vac. Sci. Technol. B 5, 1263 (1987).

${ }^{\circ}$ N. C. Giles-Taylor, R. N. Bicknell, D. K. Blanks, T. H. Myers, and J. F. Schetzina, J. Vac. Sci. Technol. A 3, 76 (1985).

J. G. Mendoza-Alvarez, J. Gonzalez-Hernandez, F. Sanchez-Sinencio, O. Zelaya, and S. S. Chao, J. Cryst. Growth 86, 391 (1988).

${ }^{8}$ A. J. Ricco, H. S. White, and M. S. Wrighton, J. Vac. Sci. Technol. A 2 , $910(1984)$.

${ }^{9}$ E. Molva, K. Saminadayar, J. L. Pautrat, and E. Ligeon, Solid State Commun. 48, 955 (1983).

${ }^{10}$ Semiconductors and Semimetals, Cadmium Telluride edited by K. Zanio (Academic, Orlando, 1978), Vol. 13

' D. J. Olego, J. P. Faurie, S. Sivananthan, and P. M. Raccah, Appl. Phys. Lett. 47, 1172 (1985) 
12 J. H. Dinan and S. B. Qadri, J. Vac. Sci. Technol. A 3, 851 (1985). ${ }^{13}$ E. Cohen, R. A. Street, and A. Muranevich, Phys. Rev. B 28, 7115 (1983).

${ }^{14}$ H. A. Mar and N. Salanskg, J. Appl. Phys. 56, 2369 (1984)

${ }^{15}$ V. N. Babentsov, S. I. Gorban, E. A. Salkov, and N. I. Torbaev,

${ }^{16}$ C. Mikkelsen and J. B. Boyce, Phys. Rev. Lett. 49, 1412 (1982).

${ }^{17}$ Z. C. Feng, A. Mascarenhas, and W. J. Choyke, J. Lumin. 35, 329 (1986).

18 J. Garcia-Garcia, J. Gonzalez-Hernandez, Elias Lopez-Cruz, G. Contreras and J. G. Mendoza-Alvarez, J. Appl. Phys. 67, 3810 (1990).

${ }^{19}$ S. Suga, K. Cho, P. Hiesinger, and T. Koda, J. Lumin. 12/13, 109 (1976).

${ }^{20}$ R. E. Halsted and M. Aven, Phys. Rev. Lett. 14, 64 (1965)

${ }^{21}$ J. L. Pautrat, J. M. Francou, N. Magnea, E. Molva, and K. Saminadayar,
J. Cryst. Growth 72, 194 (1985)

${ }^{22}$ R. L. Harper, S. Hwang, N. C. Giles, and J. F. Schetzina, Appl. Phys. Lett. 54, 170 (1989).

${ }^{23}$ B. Monemar and E. Molva, Phys. Rev. B 32, 6554 (1985).

${ }^{24}$ S. Seto, A. Tanaka, Y. Masa, S. Dairaku, and M. Kawashima, Appl. Phys. Lett. 53, 1524 (1989)

${ }^{25}$ A. Sher, An-Ban Chen, W. E. Spicer, and C.-K. Shih, J. Vac. Sci. Technol. A 3, 112 (1985)

${ }^{26}$ S. L. Bell and S. Sen, J. Vac. Sci. Technol. A 3, 112 (1985).

${ }^{27}$ N. C. Giles, R. N. Bicknell, and J. F. Schetzina, J. Vac. Sci. Technol. A 5, 3064 (1987).

${ }^{28}$ P. B. Klein, J. Comas, and S. G. Bishop, J. Appl. Phys. 50, 4327 (1979). 